\title{
Clinical significance of serum
} pneumonitis

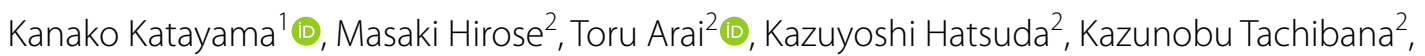
Reiko Sugawara ${ }^{1}$, Chikatoshi Sugimoto ${ }^{2}$, Takahiko Kasai ${ }^{3}$, Masanori Akira ${ }^{4}$ and Yoshikazu Inoue ${ }^{2^{*}}(\mathbb{D}$

\begin{abstract}
Background: Anti-granulocyte-macrophage colony-stimulating factor autoantibody (GMAb) has been recognized as a diagnostic biomarker for autoimmune pulmonary alveolar proteinosis (aPAP). The aims of this study were to know the incidence of increased level of serum GMAb in granulomatous lung diseases (sarcoidosis and hypersensitivity pneumonitis [HP]) and to clarify the role of GMAb. Consecutive individuals diagnosed with sarcoidosis $(n=92)$ and HP $(n=45)$ at National Hospital Organization Kinki-Chuo Chest Medical Center were retrospectively analyzed. We measured serum GMAb levels at the diagnosis. Cut-off values of GMAb discriminating aPAP $(n=110)$ from healthy controls $(n=31)$ were determined by receiver operating characteristic $(R O C)$ curve analysis. We compared the clinical features of sarcoidosis and HP patients with GMAb levels above the cut-off value ("Elevated-GMAb") with those of patients whose GMAb levels below the cut-off value ("Low-GMAb"). Radiological and pathological findings in elevated-GMAb patients were re-evaluated to elucidate the role of GMAb in granulomatous lung diseases.
\end{abstract}

Results: Analysis of ROC indicated a sensitivity and specificity of $100 \%$ at GMAb level of $3.33 \mu \mathrm{g} / \mathrm{mL}$ for discriminating aPAP from healthy controls (area under curve $=1.000, p<0.0001$ ). The percentages of elevated-GMAb sarcoidosis and HP patients were $5.4 \%(n=5)$ and $11.1 \%(n=5)$, respectively. The number of comorbid sarcoidosis and HP patients with aPAP was two and one, respectively. Elevated-GMAb sarcoidosis patients presented with significantly higher serum levels of Krebs von den Lungen (KL)-6, surfactant protein-D (SP-D), lactate dehydrogenase, and the requirement of systemic corticosteroid therapy. Elevated-GMAb HP patients demonstrated older age, higher serum KL-6, SP-D, carcinoembryonic antigen, and cytokeratin fragment 21-1 levels, and a higher percentage of lymphocytes in bronchoalveolar lavage than low-GMAb patients. A subset of patients presented with radiological and pathological findings characteristic of aPAP.

Conclusions: We demonstrated the percentage of elevated-GMAb sarcoidosis and HP patients who presented with several features suggestive of aPAP. Elevated-GMAb sarcoidosis and HP patients without definitive aPAP diagnosis may

\footnotetext{
*Correspondence: giichiyi@me.com; inoue.yoshikazu.nt@mail.hosp.go.jp

${ }^{2}$ Clinical Research Center, National Hospital Organization Kinki-Chuo

Chest Medical Center, Sakai City, Osaka, Japan

Full list of author information is available at the end of the article
}

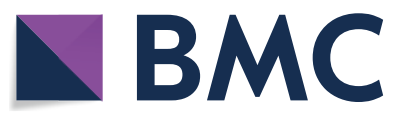

(c) The Author(s) 2020. This article is licensed under a Creative Commons Attribution 4.0 International License, which permits use, sharing, adaptation, distribution and reproduction in any medium or format, as long as you give appropriate credit to the original author(s) and the source, provide a link to the Creative Commons licence, and indicate if changes were made. The images or other third party material in this article are included in the article's Creative Commons licence, unless indicated otherwise in a credit line to the material. If material is not included in the article's Creative Commons licence and your intended use is not permitted by statutory regulation or exceeds the permitted use, you will need to obtain permission directly from the copyright holder. To view a copy of this licence, visit http://creativeco mmons.org/licenses/by/4.0/. The Creative Commons Public Domain Dedication waiver (http://creativecommons.org/publicdomain/ zero/1.0/) applies to the data made available in this article, unless otherwise stated in a credit line to the data. 
have subclinical or early-stage aPAP and may not necessarily indicate false positives. Upon diagnosis of sarcoidosis or HP, measurement of GMAb may be useful in detecting possible comorbidity of subclinical or early-onset aPAP.

Keywords: Autoantibody, Granulocyte-macrophage colony-stimulating factor, Hypersensitivity pneumonitis, Pulmonary alveolar proteinosis, Sarcoidosis

\section{Introduction}

Pulmonary alveolar proteinosis (PAP) is a rare disorder characterized by the accumulation of surfactant lipids and proteins in alveolar spaces [1-3]. Kitamura et al. detected neutralizing anti-granulocyte-macrophage colony-stimulating factor autoantibody (GMAb) in serum and bronchoalveolar lavage from autoimmune pulmonary alveolar proteinosis (aPAP) patients $[4,5]$. Insufficient maturation of pulmonary monocyte/macrophage populations in the presence of GMAb with neutralizing capacity of granulocyte-macrophage colony-stimulating factor (GM-CSF) is considered to cause aPAP $[2,3,5,6]$. In aPAP, GMAb is pathogenic and thus acts as a diagnostic biomarker. We diagnosed aPAP using our serum cut-off value of GMAb to discriminate aPAP from normal controls and control diseases $[3,7,8]$.

In antigen presentation and granuloma formation, GM-CSF plays an important role [9]. Sarcoidosis is a systemic granulomatous disorder with unknown etiology, and previous reports have suggested that its pathophysiology is an aberrant immune response driven by an unidentified antigen, and associated with macrophage/monocyte-derived cytokines such as GM-CSF [10]. Hypersensitivity pneumonitis (HP) is a granulomatous lung disease characterized by an immunological reaction to inhaled antigens [11]. It is hypothesized that environmental exposure may play a role in triggering autoimmunity in aPAP $[12,13]$. Hence, some pathophysiological link between aPAP and granulomatous diseases including sarcoidosis and HP might be present.

Recently, several aPAP patients with sarcoidosis have been reported [14-18]. Verma et al. described five patients with histopathological features of both PAP and HP [19]. Nishimura et al. [20] reported that serum GMAb levels were slightly elevated in sarcoidosis patients compared with healthy controls. Relatively elevated-GMAb levels in granulomatous lung diseases such as sarcoidosis or HP may exist, but the incidence and clinical features of this condition are unknown. This study aimed to retrospectively analyze the incidence and clinical features of elevated-GMAb levels with sarcoidosis and HP.

\section{Patients and methods}

\section{Patients}

This study included consecutive sarcoidosis $(n=92)$ and HP $(\mathrm{n}=45)$ patients whose serum samples were stored at diagnosis in the National Hospital Organization (NHO) Kinki-Chuo Chest Medical Centre between June 2003 and December 2017. Patients underwent bronchoscopy and/or surgical lung biopsy for the pathological diagnosis. Medical conditions of all patients were discussed and their diagnoses were established at our institutional multidisciplinary conferences. To determine the cut-off values to distinguish aPAP from healthy controls, this study also included consecutive individuals diagnosed with aPAP $(\mathrm{n}=110)$ in our institute between July 1997 and September 2016, and age-/ sex-matched healthy controls $(\mathrm{n}=31)$. Healthy controls had no symptoms, history of major illnesses, or abnormal shadows on chest radiography during check-up at our hospital in 2017.

\section{Diagnostic criteria of sarcoidosis, HP and aPAP}

Sarcoidosis diagnosis was based on the 2006 Diagnostic Criteria and guidelines for sarcoidosis published by the Japanese Society of Sarcoidosis and Other Granulomatous Disorders (JSSOG) [21, 22].

The diagnosis of HP was arrived at based on the following criteria [11, 23-26]: a combination of compatible symptoms (dyspnea and/or cough and/or symptoms with onset shortly after exposure and/or recurrent viral symptoms), suggestive computerized tomography (CT) findings (extensive ground glass opacities [GGOs], centrilobular nodule, peribronchovascular fibrosis, upper lobe predominant fibrosis and/or air trapping), and suggestive pathological findings (presence of granulomas, giant cells and/or mixed bronchocentric/usual interstitial pneumonia-like pattern) or compatible pathological findings (any fibrosis without a higher probability of any other disease). Patients with chronic HP were required to have pathological findings, high-resolution computed tomography (HRCT) findings, or both, indicative of fibrosis or respiratory symptoms that were present for six months or longer [23].

The diagnosis of aPAP was based on pathological and/ or cytological findings of transbronchial lung biopsy, bronchoalveolar lavage (BAL), or surgical lung biopsy 
with both HRCT appearance and GMAb level higher than 95th percentile of healthy controls according to the diagnostic criteria of the Japanese Ministry of Health, Labour and Welfare and our recent review article [3, 27].

\section{Clinical findings}

Data were extracted from medical records. Variables included demographic data, laboratory data, BAL fluid findings, and pulmonary function test results at sarcoidosis and HP diagnoses. We investigated the number of patients who received systemic corticosteroid treatment after sarcoidosis or HP diagnosis in our hospital.

\section{Measurements}

At diagnosis, GMAb levels in sera were measured by the specific enzyme-linked immunosorbent assay (ELISA) methods previously reported [2, 4, 27-29], with minor modifications to monoclonal GMAb reference standard and the regression method. The monoclonal antibody used for the standard was kindly provided by EVEC, Inc. (Sapporo, Japan). We measured serum GMAb at diagnosis and follow-up latest point in GMAb-positive sarcoidosis and HP patients whose serum samples were obtained after diagnosis. In complicated cases of sarcoidosis or HP with aPAP, GMAb levels were measured at diagnosis of sarcoidosis or HP. We measured serum levels of Krebs von den Lungen (KL)-6 and surfactant protein-D (SP-D) using commercially available ELISA kits (ED046: Eisai Co., Tokyo, Japan; SP-D kit: Yamasa Co., Tokyo, Japan). Serum KL-6 and SP-D cut-off values were $500 \mathrm{U} / \mathrm{mL}$ and $110 \mathrm{ng} / \mathrm{mL}$, respectively [2].

\section{Radiological and pathological evaluation}

We re-evaluated radiological and pathological findings related to PAP in elevated-GMAb sarcoidosis and HP patients without complication of aPAP. All re-evaluated HRCT scans and biopsies were performed at sarcoidosis or HP diagnosis. The features on HRCT scans suggestive of PAP were GGO, interlobular septal thickening, crazing paving pattern, consolidation, geographic distribution, and subpleural sparing. Those potentially accompanying PAP were defined by traction bronchiectasis, cysts, and honeycombing. All HRCTs were reviewed and scored by a senior chest radiologist (M. A.) who was blinded to clinical information. The histologic features suggestive of PAP were eosinophilic granular materials, periodic acid-Schiff (PAS) staining-positive materials, and surfactant protein-A (SP-A) positive materials. Those potentially accompanying PAP were defined by foamy macrophages, a hyaline globule/sclerotic central core, lymphocytic infiltration, and interstitial fibrosis. Negative findings for PAP included neoplastic lesions, granulomatous lesions, neutrophilic/eosinophilic infiltration, and necrosis. All surgical lung biopsy and/or transbronchial lung biopsy slides were reviewed and scored by a senior pulmonary pathologist (T.K.) who was blinded to clinical information.

\section{Receiver operating characteristic (ROC) curve analysis}

We performed ROC curve analysis for differentiating aPAP from the following: (1) healthy control, (2) sarcoidosis and HP patients, and (3) healthy controls, sarcoidosis, and HP patients. We used the Youden's index for analysis. We defined patients with GMAb levels at or above the cut-off value as elevated-GMAb patients and those with GMAb levels below the cut-off value as lowGMAb patients.

\section{Statistical analysis}

Continuous variables were reported as the median (interquartile range [IQR]) and were compared using Wilcoxon singed-rank test. Categorical variables were reported as counts (percentages) and were compared using Fisher's exact test. A $p$-value $<0.05$ was considered statistically significant. All data analyses were conducted using JMP ${ }^{\circledR}$ 13 (SAS Institute Inc., Cary, NC, USA).

\section{Results}

GMAb cut-off values for discriminating aPAP from healthy controls, sarcoidosis and HP patients, and both

Of the 92 sarcoidosis patients, two (2.1\%) were complicated by aPAP. Among the 45 HP patients, one $(2.2 \%$, chronic HP) was complicated by aPAP, 39 (87\%) had chronic HP, and 30 (67\%) required surgical lung biopsy for diagnosis. The median follow-up period was 5.6 years (Interquartile range [IQR]: $3.6,10.2$ ) in sarcoidosis patients and 4.9 years (IQR: 2.4, 7.3) in HP patients. Patients' clinical characteristics are shown in Table 1.

Analysis of ROC indicated a sensitivity and specificity of $100 \%$ at GMAb level of $3.33 \mu \mathrm{g} / \mathrm{mL}$ for discriminating aPAP from healthy controls (Area under the curve $[\mathrm{AUC}]=1.000, p<0.0001$ ) (Fig. 1 ). We defined elevatedGMAb patients as those with GMAb levels $\geq 3.33 \mu \mathrm{g} / \mathrm{mL}$. Analysis of ROC for discriminating aPAP from sarcoidosis and HP (AUC $=0.987, p<0.0001)$ indicated a sensitivity of $100 \%$ and specificity of $94.8 \%$ at a cut-off value of $3.33 \mu \mathrm{g} / \mathrm{mL}$ for GMAb levels. Further, ROC analysis indicated a sensitivity of $100 \%$ and specificity of $95.8 \%$ at a cut-off GMAb value of $3.33 \mu \mathrm{g} / \mathrm{mL}$ for discriminating aPAP from both healthy controls and sarcoidosis and HP patients $(\mathrm{AUC}=0.989, p<0.0001)$.

Serum GMAb levels in aPAP were significantly higher than those in healthy controls and sarcoidosis and HP patients (Fig. 2). 
Table 1 Clinical characteristics of patients

\begin{tabular}{|c|c|c|c|c|}
\hline Variables & Healthy controls & aPAP & Sarcoidosis & $\mathrm{HP}$ \\
\hline $\mathrm{N}$ & 31 & 110 & 92 & 45 \\
\hline Age, [IQR] years & $56[55,59]$ & $56[46,65]$ & $59[38,70]$ & $61[53,70]$ \\
\hline Male, $\mathrm{n}[\%]$ & 15 [48.3] & $77[70]$ & $39[42.3]$ & $24[53.3]$ \\
\hline Complication of aPAP, n [\%] & - & - & $2[2.1]$ & $1[2.2]$ \\
\hline $\mathrm{GMAb}[\mathrm{IQR}] \mu \mathrm{g} / \mathrm{mL}$ & $0.85[0.52,1.06]$ & $40.5[17.1,71.8]$ & $0.43[0.16,0.85]$ & $0.87[0.54,1.10]$ \\
\hline
\end{tabular}

Data are expressed as the median [IQR] or number [\%]

aPAP autoimmune pulmonary alveolar proteinosis, HP hypersensitivity pneumonitis, GMAb anti-granulocyte-macrophage colony-stimulating factor autoantibody

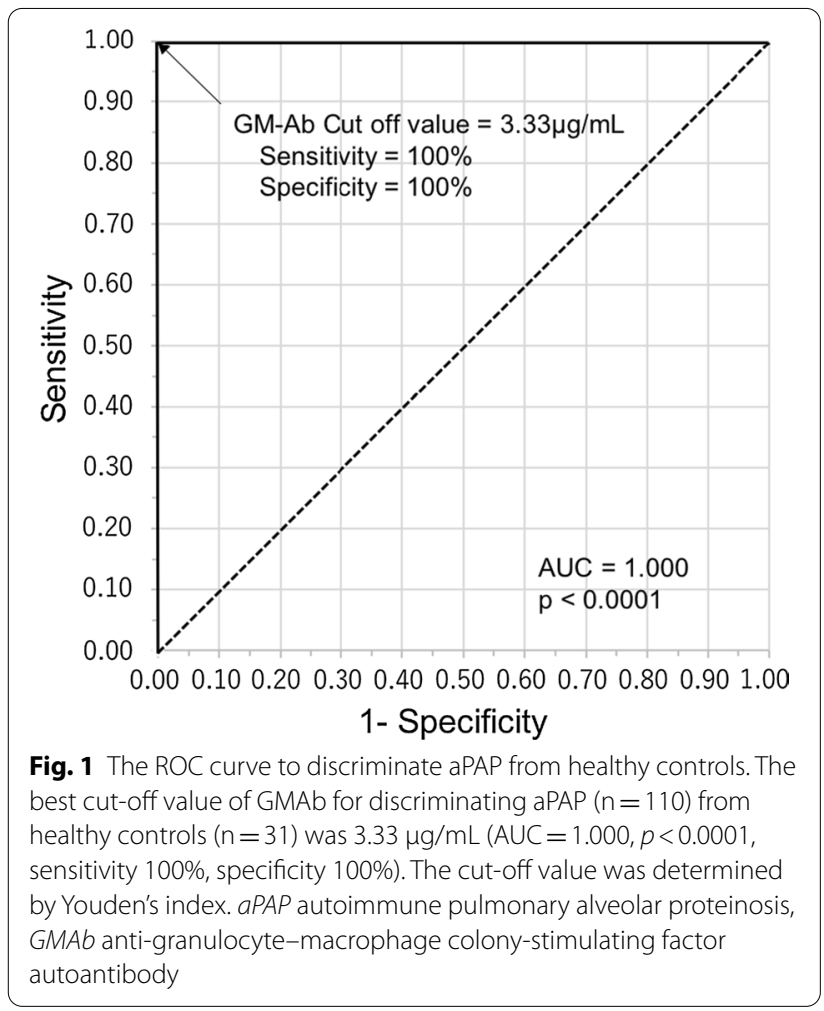

Comparison of clinical features between patients with low-GMAb and elevated-GMAb sarcoidosis and HP

The number of elevated-GMAb sarcoidosis patients, including comorbid patients with aPAP $(n=2)$, was five (5.4\%). The number of elevated-GMAb HP patients, including a comorbid patient with aPAP $(n=1)$, was five (11.1\%). We also measured the serum GMAb levels of the most recent samples obtained after diagnosis from the four elevated-GMAb patients (two sarcoidosis and two HP patients) without complication of aPAP. The GMAb levels of all four patients remained higher than the cutoff values (Fig. 3). The median interval between measurements of GMAb levels was 4.4 years (IQR: 1.1, 7.8).

Clinical characteristics of 92 sarcoidosis patients, grouped according to elevated- or low-GMAb status, are shown in Table 2. Elevated-GMAb sarcoidosis patients presented with higher lactate dehydrogenase $(\mathrm{LDH})(p=0.035), \mathrm{KL}-6(p=0.003)$, and SP-D $(p=0.022)$ levels than those of low-GMAb patients. More sarcoidosis patients with elevated-GMAb required systemic corticosteroid therapy after diagnosis than patients with low-GMAb $(p=0.002)$. ElevatedGMAb sarcoidosis patients without aPAP complication $(\mathrm{n}=3)$ presented with higher KL-6 levels than those of low-GMAb patients $(\mathrm{n}=87)(p=0.049)$. Sarcoidosis patients with elevated-GMAb and without aPAP complication also required systemic corticosteroid therapy after diagnosis $(n=2,66.7 \%)$ more frequently than lowGMAb patients $(\mathrm{n}=10,11.4 \%)(p=0.048)$.

Characteristics of $45 \mathrm{HP}$ patients, grouped according to elevated- or low-GMAb status, are shown in Table 3. Patients with elevated-GMAb HP presented with older age $(p=0.039)$, higher lymphocyte counts in BAL $(p=0.019)$, and higher serum levels of KL-6 $(p=0.001)$, SP-D $(p=0.004)$, carcinoembryonic antigen (CEA) $(p=0.023)$, and cytokeratin fragment 21-1 (CYFRA) $(p=0.001)$ than did patients with low-GMAb HP. Whereas elevated-GMAb HP patients without aPAP complication $(\mathrm{n}=4)$ presented with higher lymphocyte counts in BAL $(p=0.016)$, and higher serum levels of KL-6 $(p=0.003)$, and SP-D $(p=0.007)$ than those of low-GMAb subjects $(n=40)$.

\section{Radiological and pathological re-evaluation in patients with elevated-GMAb sarcoidosis and HP patients}

The radiological and pathological re-evaluation of findings related to PAP in elevated-GMAb sarcoidosis and HP patients without aPAP diagnosis is shown in Table 4. In elevated-GMAb sarcoidosis patients without aPAP, GGO (2 of 3 patients) and interlobular septal thickening ( 1 of 3 patients) were observed on CT scans. Two elevated-GMAb HP patients without aPAP presented with eosinophilic granular material, and two patients presented with foamy cells within lung alveoli. 


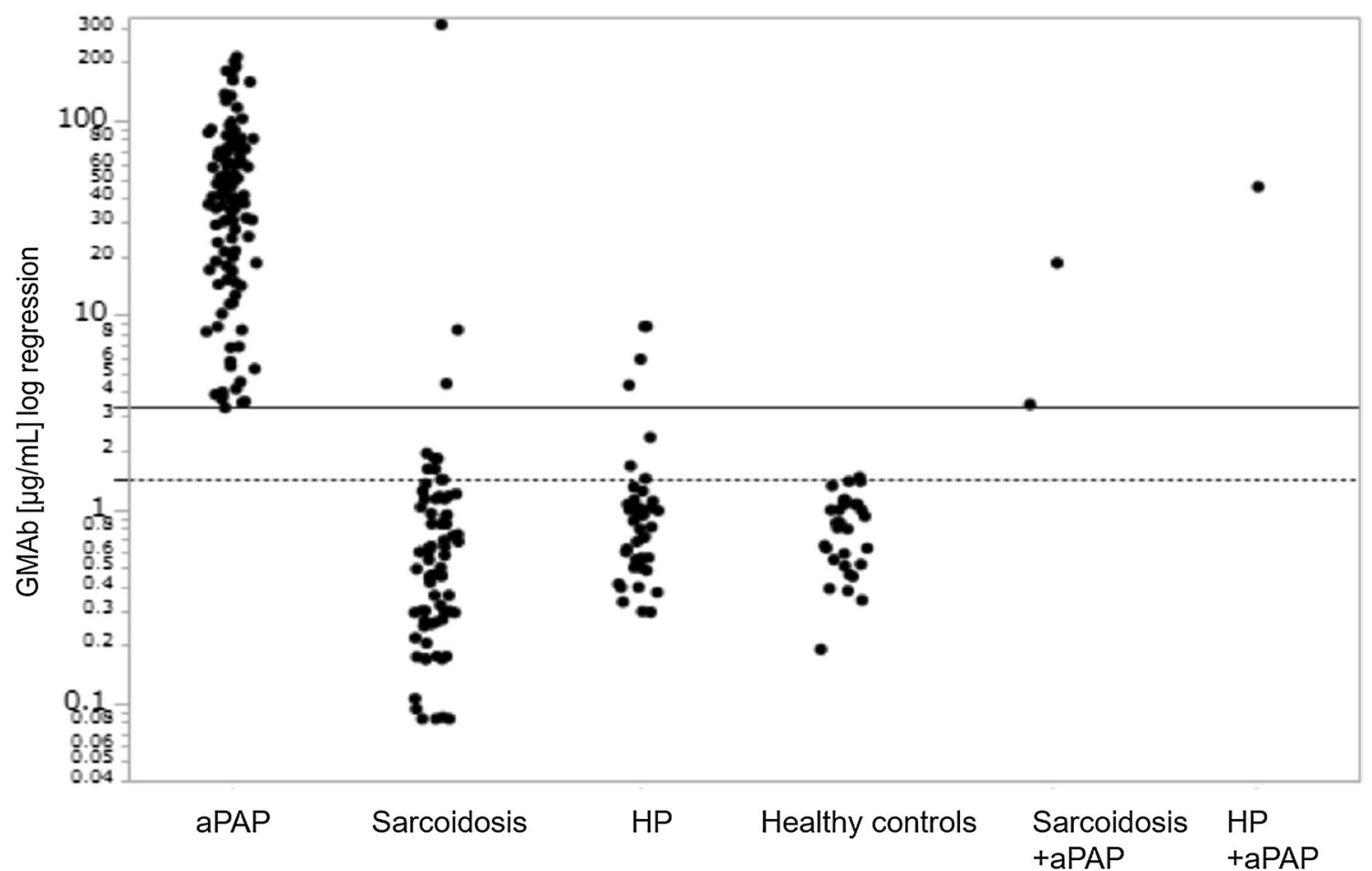

Fig. 2 GMAb levels in serum. GMAb levels in serum of aPAP $(n=110)$, sarcoidosis $(n=90), \operatorname{HP}(n=44)$, healthy controls $(n=31)$, sarcoidosis with complication of aPAP $(n=2)$ and HP with complication of aPAP $(n=1)$ patients. Horizontal dotted line shows the 95th percentile value for healthy controls $(1.42 \mu \mathrm{g} / \mathrm{mL})$ and horizontal solid line shows cut-off value $(3.33 \mu \mathrm{g} / \mathrm{mL})$ for discriminating aPAP from healthy controls. GMAb anti-granulocyte-macrophage colony-stimulating factor autoantibody, aPAP autoimmune pulmonary alveolar proteinosis, HP hypersensitivity pneumonitis

\section{Discussion}

To the best of our knowledge, this is the first study to clarify the incidence and clinical features of patients with elevated-GMAb sarcoidosis and HP. The percentages of elevated-GMAb sarcoidosis and HP patients were $5.4 \%$ and $11.1 \%$, respectively. Of the five elevatedGMAb sarcoidosis patients, two were complicated with aPAP. Of the five elevated-GMAb HP patients, one was complicated with aPAP. Significantly higher serum levels of LDH, KL-6, and SP-D were noted in patients with elevated-GMAb sarcoidosis than in patients with lowGMAb sarcoidosis. Even in elevated-GMAb HP patients, significantly higher serum levels of KL-6, SP-D, CEA, CYFRA, and higher lymphocyte count in BAL were observed than in low-GMAb patients. These clinical features observed in elevated-GMAb sarcoidosis and HP patients are considered characteristic of patients with aPAP. Further, elevated-GMAb sarcoidosis and HP patients without aPAP complication presented with some radiological and pathological features characteristic of aPAP. We diagnosed patients with aPAP if both pathological and radiological findings were consistent with the features of PAP, in addition to having GMAb levels higher than 95th percentile of healthy controls. Elevated-GMAb sarcoidosis and HP patients without aPAP complication did not meet all these criteria and were not diagnosed with aPAP before the present study; however, subclinical aPAP or early-stage aPAP was suspected.

There are several reports on GMAb cut-off values for aPAP diagnosis. Using polyclonal GMAb as the standard, Nishimura et al. [20] and Nakata et al. [29] reported cutoff values of $2.8 \mu \mathrm{g} / \mathrm{mL}$ and $1.0 \mu \mathrm{g} / \mathrm{mL}$ for GMAb, respectively. By comparison, using monoclonal GMAb as the standard, we reported a cut-off value of $0.5 \mu \mathrm{g} / \mathrm{mL}$ [2], whereas Uchida et al. considered $5.0 \mu \mathrm{g} / \mathrm{mL}$ as the cut-off value [27]. The cut-off value $(3.33 \mu \mathrm{g} / \mathrm{mL})$ that we reassessed in this cohort study using monoclonal GMAb was appropriate compared to previous GMAb reports.

Most of the previous studies have reported GMAb cut-off values for both aPAP and healthy controls $[6,27]$. Recently, Nakata et al. validated a new serum GMAb testing kit and provided an optimal cut-off value for these individuals [29]. Several studies have evaluated GMAb levels in other lung diseases $[5,8,20]$. Nishimura et al. 


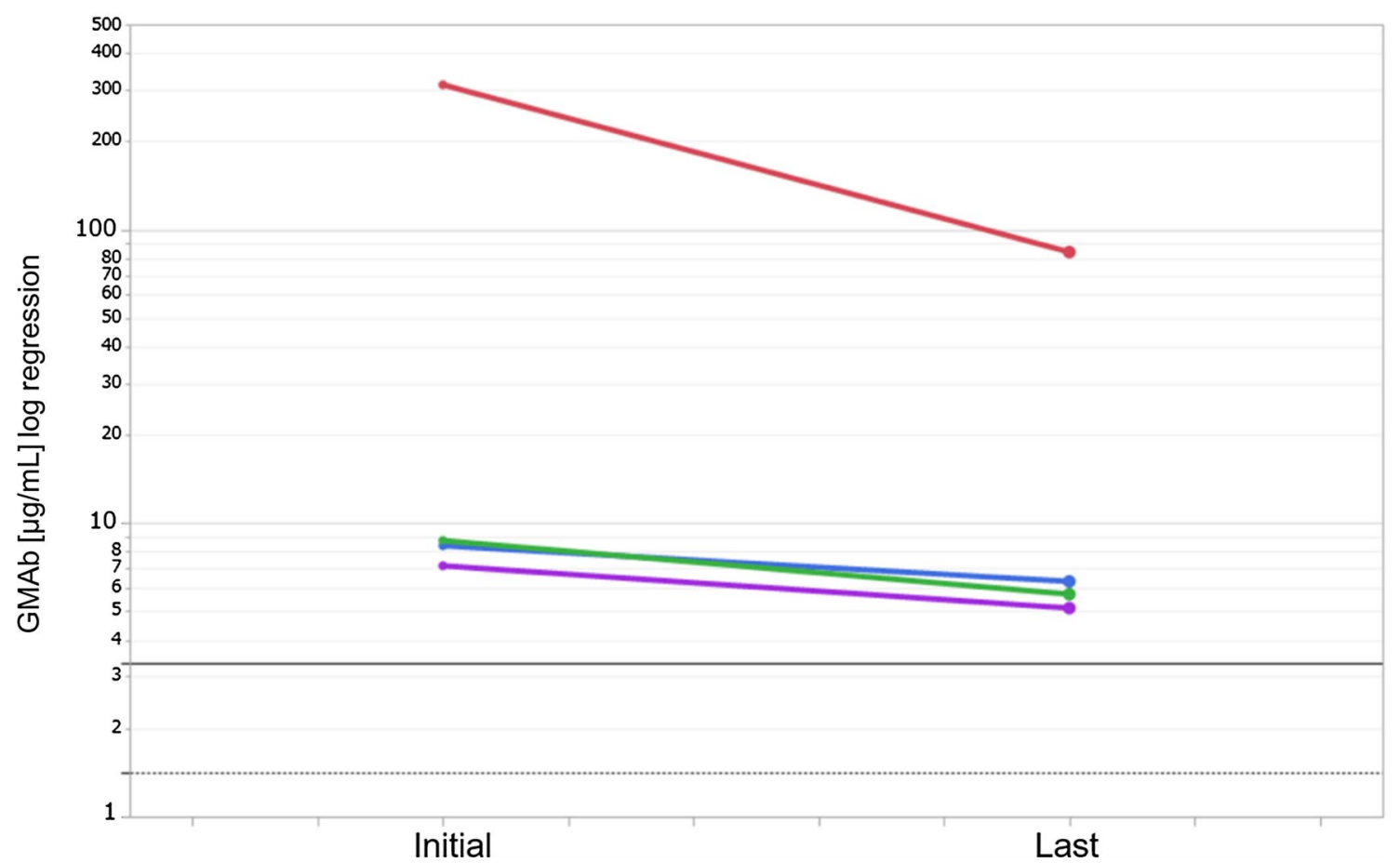

Fig. 3 Changes in GMAb levels in four elevated-GMAb patients without the complication of aPAP. We measured serum GMAb at diagnosis and follow-up latest point in elevated-GMAb sarcoidosis $(n=2)$ and HP $(n=2)$ patients whose serum samples were obtained after diagnosis. Data at "initial" and "last" are the GMAb levels at the time of diagnosis and most recently after diagnosis, respectively. The median interval between measurements of GMAb levels was 4.4 years (IQR: 1.1, 7.8). Horizontal black dotted line shows the 95th percentile value for healthy controls $(1.42 \mu \mathrm{g} / \mathrm{mL})$ and horizontal black solid line shows cut-off value $(3.33 \mu \mathrm{g} / \mathrm{mL})$ for discriminating aPAP from healthy controls. GMAb anti-granulocytemacrophage colony-stimulating factor autoantibody, aPAP autoimmune pulmonary alveolar proteinosis, HP hypersensitivity pneumonitis, IQR interquartile range

demonstrated the cut-off serum GMAb values measured by ELISA to discriminate aPAP from healthy controls or other lung diseases as $2.0 \mu \mathrm{g} / \mathrm{mL}$ (sensitivity $100 \%$, specificity $100 \%$ ) or $2.8 \mu \mathrm{g} / \mathrm{mL}$ (sensitivity $100 \%$, specificity 98\%) [20]. They reported that GMAb levels in sarcoidosis and pneumoconiosis patients were slightly higher than those in healthy controls [20]. In our study, some sarcoidosis and HP patients showed higher GMAb levels than healthy controls. We conducted ROC curve analysis for differentiating aPAP from the following combinations: (1) healthy controls, (2) sarcoidosis and HP patients, and (3) healthy controls, sarcoidosis, and HP patients. The GMAb cut-off value was $3.33 \mu \mathrm{g} / \mathrm{mL}$ for discrimination by aPAP for all the three aforementioned combinations.

Several aPAP cases complicated with sarcoidosis have been reported [14-17]. Environmental exposure to microbial agents, including Mycobacterium and Propionibacterium acnes, may be the causative factors of sarcoidosis [30, 31]. Infiltration of macrophages, T-cells, and cytokines such as GM-CSF results in granuloma formation [30, 31]. Patients with aPAP are immunocompromised, as macrophages and neutrophils become dysfunctional due to GMAb [32, 33]. They are susceptible to infection and could present with sarcoidosis as a result of exposure to microbial agents. However, sarcoidosis preceding aPAP has also been reported $[17,18]$. Akasaka et al. reported that corticosteroid therapy may worsen the disease severity in aPAP patients because corticosteroids suppress the function of macrophages [34]. In patients with sarcoidosis preceding aPAP, corticosteroid therapy for sarcoidosis could worsen early-stage or subclinical aPAP and result in aPAP complications. Verma et al. reported five patients with histopathological features of PAP and HP on surgical lung biopsy, although they did not measure GMAb [19]. Environmental factors may trigger aPAP as well as HP [13]. Infection in aPAP patients could be related to HP pathogenesis. On the contrary, in HP, lipoproteinaceous materials could accumulate after increased leakage to the alveolar spaces. Further studies are required to clarify these possibilities and whether sarcoidosis/HP or aPAP preceded the other, or if they occurred simultaneously.

Measuring GMAb values at the diagnosis of sarcoidosis or HP is desirable to enable detection of coexisting 
Table 2 Comparison of clinical features of low- and elevated-GMAb sarcoidosis patients

\begin{tabular}{|c|c|c|c|}
\hline Characteristics & Low-GMAb $(n=87)$ & Elevated-GMAb $(n=5)$ & $p$-value \\
\hline Age, years & $58[38,70]$ & $60[46,64]$ & 0.849 \\
\hline Male, $\mathrm{n}$ & $37[42.5]$ & $2[40]$ & 1.000 \\
\hline BAL Ly, \% & $27.6[19,40.8]$ & $51.5[33.6,71.6]$ & 0.073 \\
\hline BAL CD4/CD8 & $7.1[0.82,7.83]$ & $7.1[3.8,8.2]$ & 0.723 \\
\hline $\mathrm{LDH}, \mathrm{U} / \mathrm{L}$ & 185 [159-205] & $368[321-414]$ & 0.035 \\
\hline $\mathrm{KL}-6, \mathrm{U} / \mathrm{mL}$ & $352[242,581]$ & $2318[871,9608]$ & 0.003 \\
\hline $\mathrm{SP}-\mathrm{D}, \mathrm{ng} / \mathrm{mL}$ & $64[33,115.7]$ & $244[80,882]$ & 0.022 \\
\hline $\mathrm{SP}-\mathrm{A}, \mathrm{ng} / \mathrm{mL}$ & $31.2[24.6,47.5]$ & $47.2[21.2,135]$ & 0.475 \\
\hline CEA, ng/mL & $2.2[1.4-3.1]$ & $5.2[1.9-8.5]$ & 0.444 \\
\hline CYFRA, ng/mL & $1.9[1.2-2.8]$ & $8.1[5.4-10.7]$ & 0.090 \\
\hline $\mathrm{ACE}, \mathrm{U} / \mathrm{L}$ & $21.8[16.8,28.3]$ & $31.3[17.5,40.8]$ & 0.146 \\
\hline $\mathrm{sIL}-2 \mathrm{R}, \mathrm{U} / \mathrm{mL}$ & $812[522,1380]$ & $954[608,2011]$ & 0.518 \\
\hline$\% F V C, \%$ & $101.3[83.8,114.4]$ & $81.1[76.2,110]$ & 0.406 \\
\hline$\% D L c o, \%$ & $79.5[63.8,97.3]$ & $53.5[34.7,103]$ & 0.334 \\
\hline Extra pulmonary lesions, $n$ & $47[54.0]$ & $4[80]$ & 0.376 \\
\hline Eye, $n$ & 28 [32.2] & $3[60]$ & \\
\hline Skin, $n$ & $6[6.9]$ & $1[20]$ & \\
\hline Heart, n & $5[5.7]$ & $1[33.3]$ & \\
\hline Others, $\mathrm{n}$ & 9 [10.3] & $1[33.3]$ & \\
\hline Use of steroids, $n$ & $10[11.5]$ & $4[80]$ & 0.002 \\
\hline
\end{tabular}

Data are expressed as the median [IQR] or number [\%]

$G M A b$ anti-granulocyte-macrophage colony-stimulating factor autoantibody, $B A L$ bronchial alveolar lavage, $L y$ lymphocytes, $L D H$ lactate dehydrogenase, $K L-6$ Krebs von den Lungen-6, SP-D surfactant protein-D, SP-A surfactant protein-A, CEA carcinoembryonic antigen, CYFRA cytokeratin fragment 21-1, ACE angiotensin-converting enzyme, slL-2R soluble interleukin-2 receptor, \%FVC percent predicted forced vital capacity, \%DLco percent predicted carbon monoxide diffusing capacity

Table 3 Comparison of clinical features of low- and elevated-GMAb HP patients

\begin{tabular}{|c|c|c|c|}
\hline Characteristics & Low-GMAb $(n=40)$ & Elevated-GMAb $(n=5)$ & $p$-value \\
\hline Age, years & 59.5 [50.5-68.5] & 71 [64.5-73.5] & 0.039 \\
\hline Male, n & $23[57.5]$ & $2[40]$ & 0.652 \\
\hline Chronic HP, n & 35 [87.5] & $5[100]$ & 1.000 \\
\hline Trichosporon asahii, $\mathrm{n}$ & $5[14.7]$ & $1[20]$ & 1.000 \\
\hline$B A L L y, \%$ & $17.4[8.1-79.9]$ & $69.9[47.8-81.1]$ & 0.019 \\
\hline BAL CD4/CD8 & 1.7 [0.62-3.95] & $2.1[1.1-8.6]$ & 0.437 \\
\hline $\mathrm{LDH}, \mathrm{U} / \mathrm{L}$ & 208 [196-258] & 236 [211-327] & 0.213 \\
\hline $\mathrm{KL}-6, \mathrm{U} / \mathrm{mL}$ & 1416 [797-2457] & 4638 [3430-9674] & 0.001 \\
\hline SP-D, ng/mL & 180 [102-339] & 491 [330-689] & 0.004 \\
\hline SP-A, ng/mL & 59.5 [40.1-96.3] & $69.3[51.0-150]$ & 0.426 \\
\hline CEA, ng/mL & $3.7[2.4-6.2]$ & $6.1[5.6-16.1]$ & 0.023 \\
\hline CYFRA, ng/mL & $2.8[2.3-4.4]$ & $3.7[2.9-5.3]$ & 0.001 \\
\hline$\% F V C, \%$ & $83.1[64.2-90.1]$ & 95.5 [82.4-116.9] & 0.108 \\
\hline$\%$ DLco, $\%$ & $64.3[55.1-79.1]$ & 74.6 [56.6-79.5] & 0.627 \\
\hline Use of steroids, n & $15[37.5]$ & $4[80]$ & 0.146 \\
\hline
\end{tabular}

Data are expressed as the median [IQR] or number [\%]

$H P$ hypersensitivity pneumonitis, GMAb anti-granulocyte-macrophage colony-stimulating factor autoantibody, $B A L$ bronchial alveolar lavage, $L y$ lymphocytes, $L D H$ lactate dehydrogenase, $K L-6$ Krebs von den Lungen- 6 , SP-D surfactant protein-D, SP-A surfactant protein-A, CEA carcinoembryonic antigen, CYFRA cytokeratin fragment $21-1, \% F V C$ percent predicted forced vital capacity, \%DLco percent predicted carbon monoxide diffusing capacity 
Table 4 Radiological and pathological assessment in elevated-GMAb sarcoidosis and HP patients (GMAb $\geqq 3.33 \mu \mathrm{g} / \mathrm{mL}$ ) without aPAP complication

\begin{tabular}{|c|c|c|c|c|c|c|c|}
\hline \multirow{2}{*}{$\begin{array}{l}\text { Disease } \\
\mathrm{GMAb}(\mu \mathrm{g} / \mathrm{mL})\end{array}$} & \multicolumn{3}{|c|}{ Sarcoidosis $(n=3)$} & \multicolumn{4}{|c|}{$\mathrm{HP}(n=4)$} \\
\hline & 4.43 & 6.33 & 311 & 4.34 & 7.16 & 8.72 & 8.72 \\
\hline \multicolumn{8}{|l|}{ Radiological findings } \\
\hline $\mathrm{GGO}$ & + & + & - & + & + & + & + \\
\hline Interlobular septal thickening & + & - & - & - & - & - & - \\
\hline Crazing paving pattern & - & - & - & - & - & - & - \\
\hline Consolidation & - & - & - & - & - & - & - \\
\hline Geographic distribution & - & - & - & - & - & - & - \\
\hline Subpleural sparing & - & - & - & - & - & - & - \\
\hline Traction bronchiectasis & + & - & - & + & + & + & + \\
\hline Cyst & - & + & - & + & + & + & - \\
\hline Honeycombing & - & - & - & - & - & - & - \\
\hline \multicolumn{8}{|l|}{ Pathological findings } \\
\hline Eosinophilic, granular materials & - & - & - & - & - & + & + \\
\hline PAS staining-positive materials & - & - & - & - & - & - & - \\
\hline SP-A positive materials & - & - & - & - & - & - & - \\
\hline Foamy macrophages & - & - & - & - & + & + & - \\
\hline Hyaline globule/sclerotic central core & - & - & - & - & + & + & + \\
\hline Lymphocytic infiltration & - & + & - & + & + & + & + \\
\hline Interstitial fibrosis & + & + & + & + & + & + & + \\
\hline Neoplastic lesion & - & - & - & - & - & - & - \\
\hline Granulomatous lesion & + & + & - & + & + & + & + \\
\hline Neutrophilic/eosinophilic infiltration & - & - & - & + & - & - & + \\
\hline Necrosis & - & - & - & - & - & - & - \\
\hline
\end{tabular}

Serum GMAb levels were measured at sarcoidosis or HP diagnosis

aPAP autoimmune pulmonary alveolar proteinosis, GMAb anti-granulocyte-macrophage colony-stimulating factor autoantibody, $H P$ hypersensitivity pneumonitis, GGO ground glass opacity, PAS periodic acid-Schiff, SP-A surfactant protein-A

subclinical or early-stage aPAP. In sarcoidosis, more patients with elevated-GM-Ab required systemic corticosteroid therapy than patients with low-GMAb. Two cases of aPAP complicated with sarcoidosis in this study were previously reported and also required systemic corticosteroid therapy for sarcoidosis [18]. We reduced the corticosteroid dose in two patients to prevent aPAP aggravation.

On CT, findings of HP and aPAP are often difficult to distinguish. However, aPAP complication should be considered if sarcoidosis patients have GGO or interlobular septal thickening on CT scans. Pathological re-evaluation revealed features suggestive of aPAP in HP patients. It is important to evaluate the aPAP pathology in these patients alongside GMAb measurements.

The major limitations of the current study are the small sample size and retrospective design because of the rarity of the disease and the associated comorbidity. Studies with a larger sample of patients with varying diseases and disorders are required to determine the optimal cut-off value of GMAb and the incidence of increased level of GMAb in sarcoidosis and HP.

\section{Conclusions}

We demonstrated the percentage of elevated-GMAb sarcoidosis and HP patients who presented with several features suggestive of aPAP. In elevated-GMAb sarcoidosis and HP patients without aPAP diagnosis, subclinical or early-stage aPAP might be present and may not necessarily indicate false positives. Upon diagnosis of sarcoidosis or HP, GMAb should be measured to detect the possible comorbidity of subclinical or early-onset aPAP. Further longitudinal studies are required to determine the potential prognostic role of GMAb measurement in sarcoidosis and HP patients.

\section{Abbreviations}

aPAP: Autoimmune pulmonary alveolar proteinosis; BAL: Bronchoalveolar lavage; CT: Computerized tomography; ELISA: Enzyme-linked immunosorbent assay; GGO: Ground glass opacity; GMAb: Anti-granulocyte-macrophage colony-stimulating factor autoantibody; GM-CSF: Granulocyte-macrophage colony-stimulating factor; HP: Hypersensitivity pneumonitis; HRCT: Highresolution computed tomography; PAS: Periodic acid-Schiff; ROC: Receiver operating curve; SP: Surfactant protein. 


\section{Acknowledgements}

The authors would like to thank Dr. Koh Nakata, Niigata University, for technical assistance of the measurement method for granulocyte-macrophage colony-stimulating factor autoantibody.

\section{Authors' contributions}

KK: study design, data collection, data analysis, and manuscript writing. $\mathrm{MH}$ : study design, data collection, manuscript writing. TA: conception and study design, interpretation, manuscript writing, manuscript review and editing. $\mathrm{KH}$ : performing GMAb measurements. KT: data collection, data analysis. RS: data collection, data analysis. CS: data collection, data analysis. TK: pathological examination. MA: radiological evaluation. Yl: conception and study design, interpretation, manuscript writing, critical review and study supervision. All authors read and approved the final manuscript.

\section{Funding}

This work was supported by the Japan Agency for Medical Research and Development (Grant awarded to TA, TK, MA, and Yl; Grant number: JP19ek0109268) and in part by the Ministry of Health, Labour and Welfare of Japan awarded to the study Group on Pulmonary Disorders, Scientific Research/Research on intractable diseases (grant awarded to Yl; Grant number: H29-023).

\section{Availability of data and materials}

The datasets used and/or analyzed during the current study are available from the corresponding author on reasonable request.

\section{Ethics approval and consent to participate}

This study was approved by the Institutional Review Board of the NHO KinkiChuo Chest Medical Center (KCCMC; Approval number: 735, 6 February 2020).

\section{Consent for publication}

All study participants provided written, informed consent.

\section{Competing interests}

The authors declare that they have no competing interests.

\section{Author details}

1 Department of Internal Medicine, National Hospital Organization Kinki-Chuo Chest Medical Center, Sakai City, Osaka, Japan. ${ }^{2}$ Clinical Research Center, National Hospital Organization Kinki-Chuo Chest Medical Center, Sakai City, Osaka, Japan. ${ }^{3}$ Department of Pathology, National Hospital Organization Kinki-Chuo Chest Medical Center, Sakai City, Osaka, Japan. ${ }^{4}$ Department of Radiology, National Hospital Organization Kinki-Chuo Chest Medical Center, Sakai City, Osaka, Japan.

Received: 26 June 2020 Accepted: 14 September 2020

Published online: 29 September 2020

\section{References}

1. Trapnell BC, Whitsett JA, Nakata K. Pulmonary alveolar proteinosis. N Engl J Med. 2003;349:2527-39.

2. Inoue Y, Trapnell BC, Tazawa R, Arai T, Takada T, Hizawa N, et al. Characteristics of a large cohort of patients with autoimmune pulmonary alveolar proteinosis in Japan. Am J Respir Crit Care Med. 2008;177:752-62.

3. Kumar A, Abdelmalak B, Inoue Y, Culver DA. Pulmonary alveolar proteinosis in adults: pathophysiology and clinical approach. Lancet Respir Med. 2018:6:554-65.

4. Kitamura T, Tanaka N, Watanabe J, Kanegasaki S, Yamada Y, Nakata K. Idiopathic pulmonary alveolar proteinosis as an autoimmune disease with neutralizing antibody against granulocyte/macrophage colonystimulating factor. J Exp Med. 1999:190:875-80.

5. Kitamura T, Uchida K, Tanaka N, Tsuchiya T, Watanabe J, Yamada Y, et al. Serological diagnosis of idiopathic pulmonary alveolar proteinosis. Am J Resp Crit Care Med. 2000;162:658-62.

6. Uchida K, Nakata K, Suzuki T, Luisetti M, Watanabe M, Koch DE, et al. Granulocyte/macrophage-colony-stimulating factor autoantibodies and myeloid cell immune functions in healthy subjects. Blood. 2009;113:2547-56.

7. Lin FC. Clinical significance of anti-GM-CSF antibodies in idiopathic pulmonary alveolar proteinosis. Thorax. 2006;61:528-34.

8. Bonfield TL, Russell D, Burgess S, Malur A, Kavuru MS, Thomassen MJ. Autoantibodies against granulocyte macrophage colony-stimulating factor are diagnostic for pulmonary alveolar proteinosis. Am J Resp Cell Mol Biol. 2002;27:481-6.

9. Itoh A, Yamaguchi E, Kuzumaki N, Okazaki N, Furuya K, Abe S, et al. Expression of granulocyte macrophage colony-stimulating factor mRNA by inflammatory cells in the sarcoid lung. Am J Resp Cell Mol Biol. 1990;3:245-9.

10. Patterson KC, Franek BS, Müller-Quernheim J, Sperling Al, Sweiss NJ, Niewold TB. Circulating cytokines in sarcoidosis: phenotype-specific alterations for fibrotic and non-fibrotic pulmonary disease. Cytokine. 2013;61:906-11.

11. Lacasse Y, Selman M, Costabel U, Dalphin JC, Ando M, Morell F, et al. Clinical diagnosis of hypersensitivity pneumonitis. Am J Respir Crit Care Med. 2003;168:952-8.

12. Costabel U, Nakata K. Pulmonary alveolar proteinosis associated with dust inhalation. Am J Respir Crit Care Med. 2010;181:427-8.

13. Ishii H, Tazawa R, Kaneko C, Saraya T, Inoue Y, Hamano E, et al. Clinical features of secondary pulmonary alveolar proteinosis: pre-mortem cases in Japan. Eur Respir J. 2011;37:465-8.

14. Mirchandani AS, Thomson NC. First presentation of breathlessness: pulmonary alveolar proteinosis with sarcoid. B39. Am J Respir Crit Care Med. 2010;A2948. https://doi.org/10.1164/ajrccm-conference 2010.181.1_MeetingAbstracts.A2948.

15. Boerner EB, Costabel U, Wessendorf TE, Theegarten D, Hetzel M, Drent $M$, et al. Pulmonary alveolar proteinosis: another autoimmune disease associated with sarcoidosis? Sarcoidosis Vasc Diffuse Lung Dis. 2016;33:90-4.

16. Yamasue M, Nureki S-I, Usagawa Y, Ono T, Matsumoto H, Kan T, Kadota JI. Elevated serum anti-GM-CSF antibodies before the onset of autoimmune pulmonary alveolar proteinosis in a patient with sarcoidosis and systemic sclerosis. Tohoku J Exp Med. 2017;243:77-83.

17. Tanaka Y, Shirai T, Asada K, Muramatsu A, Katsumata M, Suda T. Autoimmune pulmonary alveolar proteinosis in a patient with sarcoidosis. Clin Case Rep. 2019;7:731-4.

18. Arai T, Kasai T, Shimizu K, Kawahara K, Katayama K, Sugimoto C, et al. Autoimmune pulmonary alveolar proteinosis complicated with sarcoidosis: clinical courses and serum levels of anti-granulocytemacrophage colony-stimulating factor autoantibody. Intern Med. 2020 https://doi.org/10.2169/internalmedicine.3853-19.

19. Verma H, Nicholson AG, Kerr KM, Dempsey OJ, Gibbs AR, Campbell I, et al. Alveolar proteinosis with hypersensitivity pneumonitis: a new clinical phenotype. Respirology. 2010;15:1197-202.

20. Nishimura M, Yamaguchi E, Takahashi A, Asai N, Katsuda E, Ohta T, et al. Clinical significance of serum anti-GM-CSF autoantibody levels in autoimmune pulmonary alveolar proteinosis. Biomark Med. 2018;12:151-9.

21. Sawahata M, Sugiyama Y, Nakamura Y, Nakayama M, Mato N, Yamasawa $\mathrm{H}$, et al. Age-related and historical changes in the clinical characteristics of sarcoidosis in Japan. Respir Med. 2015;109:272-8.

22. Hiraga H, Yuwai K, Hirose M. Diagnostic standard and guidelines for sarcoidosis. Jpn J Sarcoidosis Granulomatous Disord. 2007;27:89-102.

23. Vasakova M, Morell F, Walsh S, Leslie K, Raghu G. Hypersensitivity pneumonitis: perspectives in diagnosis and management. Am J Respir Crit Care Med. 2017;196:680-9.

24. Salisbury ML, Myers JL, Belloli EA, Kazerooni EA, Martinez FJ, Flaherty KR. Diagnosis and treatment of fibrotic hypersensitivity pneumonia. Where we stand and where we need to go. Am J Respir Crit Care Med. 2017;196:690-9.

25. Morisset J, Johannson KA, Jones KD, Wolters PJ, Collard HR, Walsh SLF, et al. Identification of diagnostic criteria for chronic hypersensitivity pneumonitis: an international modified Delphi Survey. Am J Respir Crit Care Med. 2018;197:1036-44.

26. Tateishi T, Johkoh T, Sakai F, Miyazaki Y, Ogura T, Ichikado K, et al. High-resolution CT features distinguishing usual interstitial pneumonia pattern in chronic hypersensitivity pneumonitis from those with idiopathic pulmonary fibrosis. Jpn J Radiol. 2020;38:524-32. 
27. Pulmonary alveolar proteinosis. https://www.nanbyou.or.jp/wp-content/ uploads/upload_files/File/229-201704-kijyun.pdf. Accessed 16 Aug 2020. (in Japanese).

28. Tazawa R, Ueda T, Abe M, Tatsumi K, Eda R, Kondoh S, et al. Inhaled GMCSF for pulmonary alveolar proteinosis. N Engl J Med. 2019;381:923-32.

29. Nakata K, Sugi T, Kuroda K, Yoshizawa K, Takada T, Tazawa R, et al. Validation of a new serum granulocyte-macrophage colony-stimulating factor autoantibody testing kit. ERJ Open Res. 2020;6:00259-2019. https://doi. org/10.1183/23120541.00259-2019.

30. Hutchinson J. Statement on sarcoidosis. Joint Statement of the American Thoracic Society (ATS), the European Respiratory Society (ERS) and the World Association of Sarcoidosis and Other Granulomatous Disorders (WASOG) adopted by the ATS Board of Directors and by the ERS Executive Committee, February 1999. Am J Respir Crit Care Med. 1999;160:736-55.

31. Iannuzzi MC, Rybicki BA, Teirstein AS. Sarcoidosis. N Engl J Med. 2007:357:2153-65.
32. Uchida K. High-affinity autoantibodies specifically eliminate granulocytemacrophage colony-stimulating factor activity in the lungs of patients with idiopathic pulmonary alveolar proteinosis. Blood. 2003;103:1089-98.

33. Uchida K, Beck DC, Yamamoto T, Berclaz PY, Abe S, Staudt MK, et al. GMCSF autoantibodies and neutrophil dysfunction in pulmonary alveolar proteinosis. N Engl J Med. 2007;356:567-79.

34. Akasaka K, Tanaka T, Kitamura N, Ohkouchi S, Tazawa R, Takada T, et al. Outcome of corticosteroid administration in autoimmune pulmonary alveolar proteinosis: a retrospective cohort study. BMC Pulm Med. 2015;15:88.

\section{Publisher's Note}

Springer Nature remains neutral with regard to jurisdictional claims in published maps and institutional affiliations.
Ready to submit your research? Choose BMC and benefit from:

- fast, convenient online submission

- thorough peer review by experienced researchers in your field

- rapid publication on acceptance

- support for research data, including large and complex data types

- gold Open Access which fosters wider collaboration and increased citations

- maximum visibility for your research: over 100M website views per year

At BMC, research is always in progress.

Learn more biomedcentral.com/submissions 